\title{
Compressor Retrofittable Solutions in Heavy- Duty Gas Turbines for Minimum Environmental Load Reduction
}

\author{
Stefano Gino Mosele ${ }^{1}$, Tiziano Garbarino ${ }^{1}$, Andrea Schneider $^{1}$, Lorenzo Cozzi $^{2}$, Andrea \\ Arnone $^{2}$, Georgios Goinis ${ }^{3}$, and Simon Hedkvist ${ }^{3}$ \\ ${ }^{1}$ Ansaldo Energia (AEN), Product Development and Engineering, 16152 Genova, Italy \\ ${ }^{2}$ UNIFI, Industrial Engineering department (DIEF), 50139 Firenze, Italy \\ ${ }^{3}$ German Aerospace Center (DLR), Institute of Propulsion Technology, 51147 Cologne, Germany
}

\begin{abstract}
International policies aiming at keeping global warming within safety limits will strongly impact gas-fired power plants. Flexibility is going to be the key-word, hence this paper focuses on possible strategies to increase turndown capability (i.e. lowering Minimum Environmental Load (MEL)) of open and combined cycle power plants (CCPP) through retrofittable compressor service packages. In particular, the following three options have been analyzed: extra-closure of Variable Inlet Guide Vanes (IGVs), Blow-Off (BO) lines opening and inlet bleed heating. All these solutions aim at reducing the compressor outlet mass-flow rate while keeping a safe stability margin. The effect of lowering the minimum load capability by opening the BO lines has been numerically investigated through full compressor 2D throughflow analyses. Moreover, the impact on compressor performance and stability of the extra-closure of IGVs has been analyzed with the support of 3D steady-state CFD modelling. Finally, the overall performance of the power-plant has been included and discussed in order to provide plant managers with a solid starting point for a technoeconomic analysis.
\end{abstract}

\section{Introduction}

Nowadays, the energy market is facing a revolution driven by the continuous development of Renewable Energy Solutions (RES). The expansion of RES is going to be even more important after the promulgations of most recent environmental policies. Even though RES are expected to have a rapid expansion in the next decades, gas-fired power plants will keep a key role within the energy sector by supporting the grid stability and peak demand [1]. This new scenario, along with the restrictive environmental regulations imposed by several countries, requires conventional power plants to be more flexible, reliable and resilient. As it is also important to renew the already installed fossil-fuel power plants (to keep a low cost to benefit ratio), big efforts need to be carried out in the development of retrofittable service packages. Within EU-funded TURBO-REFLEX project two different solutions to lower compressor MEL operating condition have been studied: BO extraction and IGV extraclosure (XC). An additional method for MEL reduction is inlet bleed heating (also called 
Anti-Icing (AI)). It consists of recirculating a small amount of hot air from the compressor outlet to the inlet in order to increase compressor inlet temperature [2].

All these options reduce the mass-flow discharged by the compressor to the combustion chamber/turbine, aiming at decreasing the MEL. Nevertheless, from AEN experience and literature review, both $\mathrm{BO}$ extraction and IGV XC have some drawbacks that, once properly assessed, a designer should carefully consider such as rear stages stability, ice formation on the first stage blade and vane rows [2], blades vibration due to flow unsteadiness [3] and Heat Recovery Steam Generator (HRSG) temperature limit [4] [5].

On the one hand, the compressor blow-off extraction study has been performed through a calibrated throughflow (TF) model, characterized by a limited computational cost. On the other hand, a validated 3D Computational Fluid Dynamics (CFD) full compressor model has been used for the IGV XC study, to understand the impact on compressor stability. Finally, the Gas Turbine (GT) and CCPP performance have been verified by a calibrated 0D model in which the effects of $\mathrm{BO}, \mathrm{IGV} \mathrm{XC}$ and $\mathrm{AI}$ have been included.

\section{Blow-off studies}

Using the 2D TF code ACDC [6] developed at the DLR, a compressor has been modelled and simulated. Through a 'Design of Experiments' (DoE), several levels of outlet flow reduction, achieved by different distributions of $\mathrm{BO}$ extraction, have been simulated. The study has been made with standard fully closed IGV at MEL. The data has been analyzed to map correlations between the key compressor metrics: aerodynamic stability, efficiency and power consumption. The compressor under investigation has 15 stages plus IGV and OGV as well as three $\mathrm{BO}$ lines at different locations along the stages. Model creation has been done through iterative configuration of ACDC to match 3D CFD data supplied by AEN. Fig. 1 shows the streamwise distribution of the diffusion factor (DF), a metric for aerodynamic stability (lower DF is better for compressor stability), for the compressor simulated at MEL. Simulation with standard bleeding flows used at MEL is denoted as 'Reference' in the graph. BO lines are denoted as EB01, EB02, and EB03, with EB01 the most upstream (1 $\left.1^{\text {st }} \mathrm{BO}\right)$ and EB03 the closer to the compressor outlet ( $3^{\text {rd }} \mathrm{BO}$ ). Fig. 1 also shows how the DF is distributed along the compressor when $\mathrm{BO}$ valves are individually opened with respect to reference (to highlight the effect on DF of $\mathrm{BO}$ extraction, row 1 and $2 \mathrm{DF}$ are out of the y-axis range). Opening a BO line results in the DF being decreased upstream of the $\mathrm{BO}$ and increased in the rear stages. All eligible BO extraction points are upstream of stator 15 (row 31), putting this stator at highest risk when increasing the mass extraction, making it the limiting row from compressor off-design (further) capability point of view.

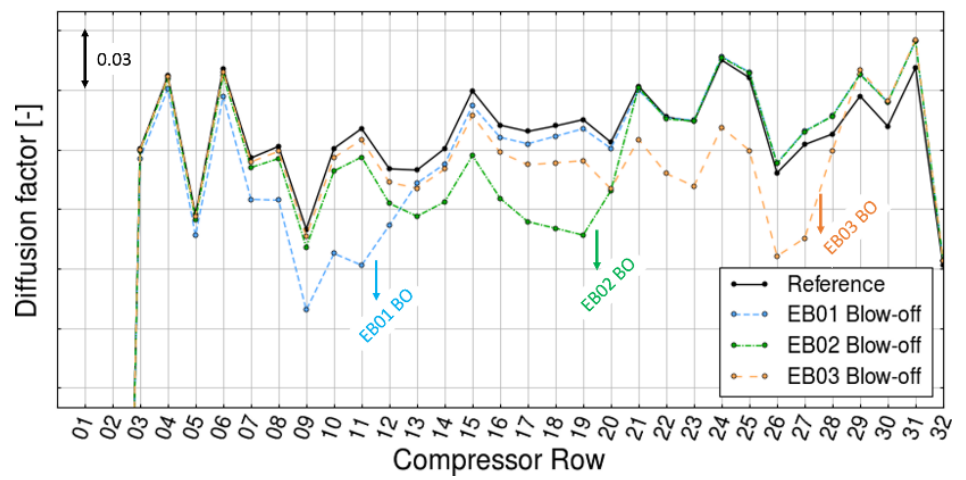

Fig. 1. Streamwise variation of the diffusion factor at MEL. Included is reference standard MEL, and $5 \%$ outlet flow reduction achieved extracting mass-flow from $\mathrm{BO}$ lines one by one. 


\subsection{Design of experiments}

The MEL BO extraction study has been set to a limit of $20 \%$ compressor outlet flow reduction from reference. The DoE has been defined and five levels of flow reduction studied. A total of 35 tests have been made with different combinations of total flow reduction and distributions between EB01, EB02 and EB03. At the lowest level, equal to the reference extracted mass-flow at standard MEL, only one combination exists. More combinations are possible at higher levels of flow reduction, with 15 tests made at $20 \%$ flow reduction. The results with maximum $\mathrm{BO}$ extraction are of chief interest, but lower levels are necessary to thoroughly map the relation between DF and increased mass-flow extracted from BO valves.

Fig. 1 showed that stator 15 (row 31) is the highest risk position for the compressor. The exact increase in DF depends on the level of flow reduction, but also the distribution between the BO lines. Fig. 2 shows a triangle plot with the delta increase in DF at stator 15 (row 31) depending on the BO mass-flow distribution for the 15 tests with $20 \%$ flow reduction. The results show that the further upstream the flow is blown-off, the smaller the increase in DF. Fig. 2 also shows the compressor efficiency and power consumption for the same combinations of BO extractions. Efficiency has a lower reduction when air is extracted further downstream instead of upstream. Despite lower efficiency, the power consumption has the same pattern as the DF, with the best values when extracting from EB01.

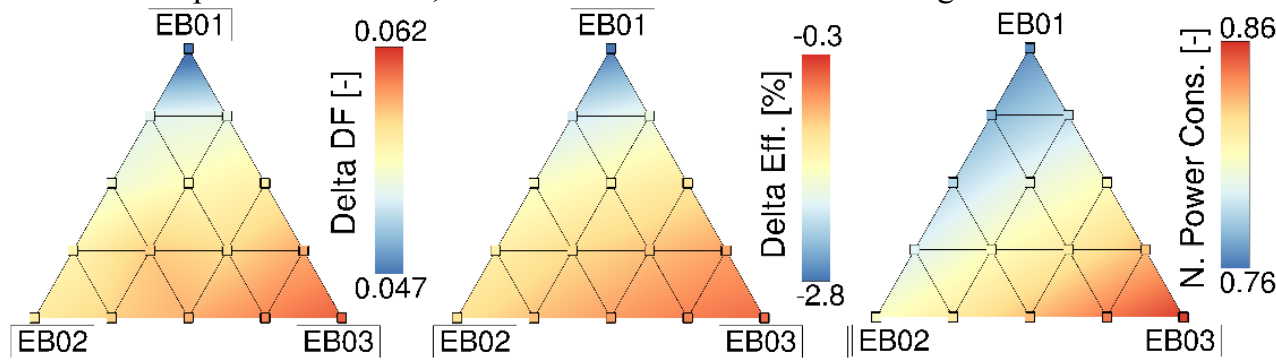

Fig. 2. Triangle graphs showing MEL delta values with respect to standard MEL in stator 15 (row 31) DF, compressor efficiency, and compressor normalized power consumption. Points show DoE result values, with color interpolation in between. Each point represents a different distribution between the three ports EB01-EB03, with the corner positions being maximum extraction from that BO line.

\section{Compressor IGV extra-closure}

One of the options available to reduce the MEL of a GT is to increase the closure of the IGV and the other stationary rows with a variable stagger configuration. This practice can significantly reduce the mass-flow elaborated and the power output of the GT but should be carefully analyzed and evaluated to assure that no stability issues will afflict the compressor. This MEL reduction strategy has been numerically investigated by the University of Florence (UNIFI). All the simulations have been performed using the CFD code TRAF [7], a flow solver for the 3D Reynolds-averaged Navier-Stokes equations. The $\mathrm{k}-\omega$ model [8] has been adopted as turbulence closure and the operating fluid has been modelled as a thermally perfect gas [9]. For all the steady analyses performed, a non-reflecting mixing plane model [10] has been used. The fluid domain has been discretized with structured grids.

The axial compressor chosen as baseline for the activities performed by UNIFI is a Fclass 15-stage plus IGV and OGV turbomachine of the AEN fleet. To regulate the elaborated mass-flow, the IGV and the stator row of the first stage have a variable stagger configuration. AEN performed an experimental campaign on the compressor, testing the machine at the current MEL operating condition and at three configurations with enhanced closure of the IGV with respect to standard MEL ranging from 2 to $4.5^{\circ}$ (TEST-24, TEST-25 and TEST- 
26). Computational grids have been generated for all the tested configurations and for another case with increased XC called RUN-27.

As shown by the example represented in Fig. 3, reporting the values of compressor inlet mass-flow and outlet total temperature, a good agreement between numerical results and experimental measurements has been obtained.
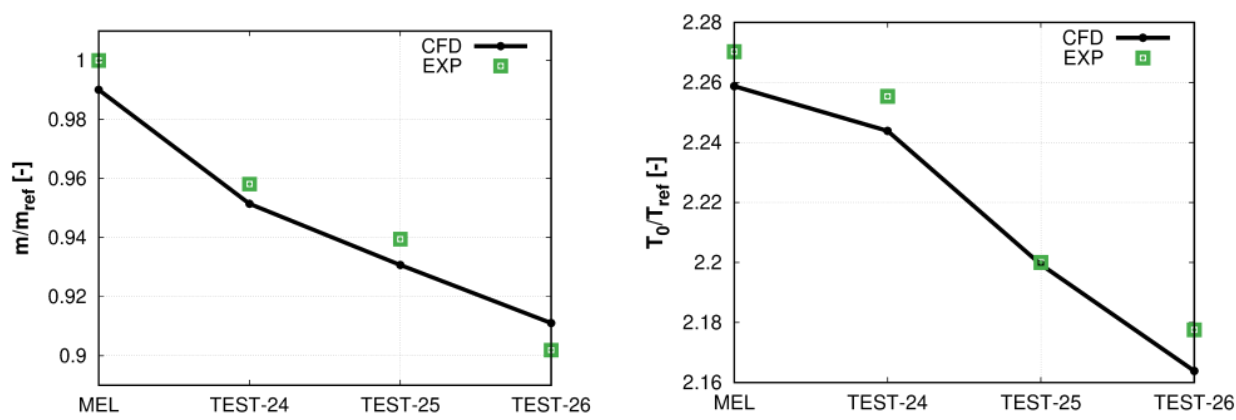

Fig. 3. Compressor inlet mass-flow and outlet total temperature for the four configurations tested

After validating the steady setup, further simulations have been performed for RUN-27 at ISO inlet conditions and very low inlet temperature, the latter condition being really challenging from a stability point of view. Extra-closing the IGVs, the first rotor row experiences an increased expansion with respect to standard MEL and hence an increased temperature drop at $\mathrm{CB} 01$ outlet section, with the risk of ice deposition on both Compressor Blade (CB)01 and Compressor Vane (CV)01. As shown in Fig. 4, at ISO conditions, both the pressure ratio $(\beta)$ of the rear stages and the loading $(\psi)$ of the last stage increase, resulting in a higher risk in terms of stability. Furthermore, lowering the inlet temperature, one can notice greater variations with higher loading on the compressor rear stages. In particular, stability issues with blade loading distributions close to stall tend to arise when a really low inlet temperature is adopted as boundary condition. Hence, it is crucial to pay attention to the ambient temperature in which the compressor operates when lowering the MEL through IGV $\mathrm{XC}$.
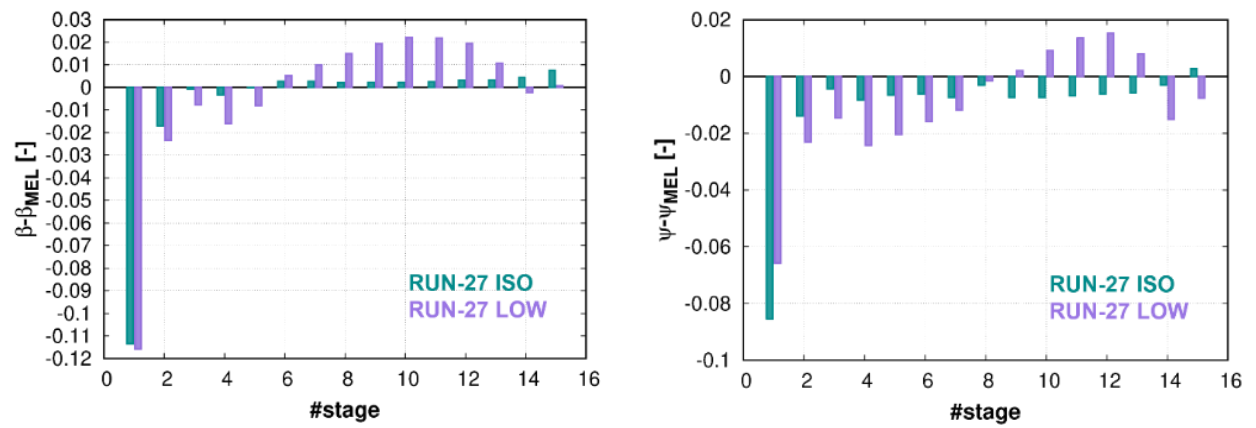

Fig. 4. Stage pressure ratio and load coefficient variations with respect to MEL for RUN-27 case

\section{MEL reduction from GT and CCPP point of view}

IGV Extra Closure, AI activation and $\mathrm{BO}$ valves regulation can be used to reduce MEL, but with different results in terms of GT power and efficiency.

A 0D AEN F-class GT model based on maps for turbomachinery and secondary air system off-design prediction has been used for simulations. The GT model has been calibrated with experimental data while the combined cycle performance data were 
calculated using a correlation between Water Steam Cycle (WSC) efficiency and GT outlet temperature. The correlation has been calibrated on real AEN plant data.

The relative efficiency delta Vs relative power delta (valid for an AEN F-class GT) is reported in Fig. 5 for different MEL reduction strategies both for GT (left) and CCPP (right). In Fig. 5 (right), the colored text boxes indicate the maximum GT outlet temperature variation (in percentage with respect to the standard MEL) for each solution. All the points are calculated at the same conditions in terms of GT setting, flame temperature (used as indicator of CO emissions) and ambient conditions (ISO, full methane). Fig. 5 (left) shows that the preferred solution for GT MEL enhancement is IGV XC (blue line) because at fixed GT power reduction the GT efficiency is higher (so the fuel saving with respect to the same standard MEL is better). The second preferred option is the $1^{\text {st }} \mathrm{BO}$ valves opening (green line) which is also the one allowing the deeper MEL reduction (depending from the BO lines geometrical features). Finally, the less efficient option is the AI activation (red line). It is reasonable to expect a linear trend in case of partial AI activation. Looking at the customer benefit, a $10 \%$ GT power reduction obtained through IGV XC saves approximately $5.4 \%$ fuel mass-flow with respect standard MEL, while BO and AI guarantee respectively $4.9 \%$ and $4.4 \%$ fuel saving. A preceding study on $\mathrm{BO}$ extraction highlighted that the cycle efficiency benefits from extracting the flow through the $1^{\text {st }} \mathrm{BO}$ with respect to the downstream ones.

$\mathrm{AI}$ and $\mathrm{BO}$ have a stronger impact on performance cycle because, differently from what happens with XC, a part of the compressed air mass-flow does not participate to the power generation (last stage blade outlet mass-flow is different than the compressor inlet one). For these simulations, it resulted that the complete compression of a quite small amount of air (AI) resulted in having more impact (=requires more power) than the partial compression of a big amount of air (BO).
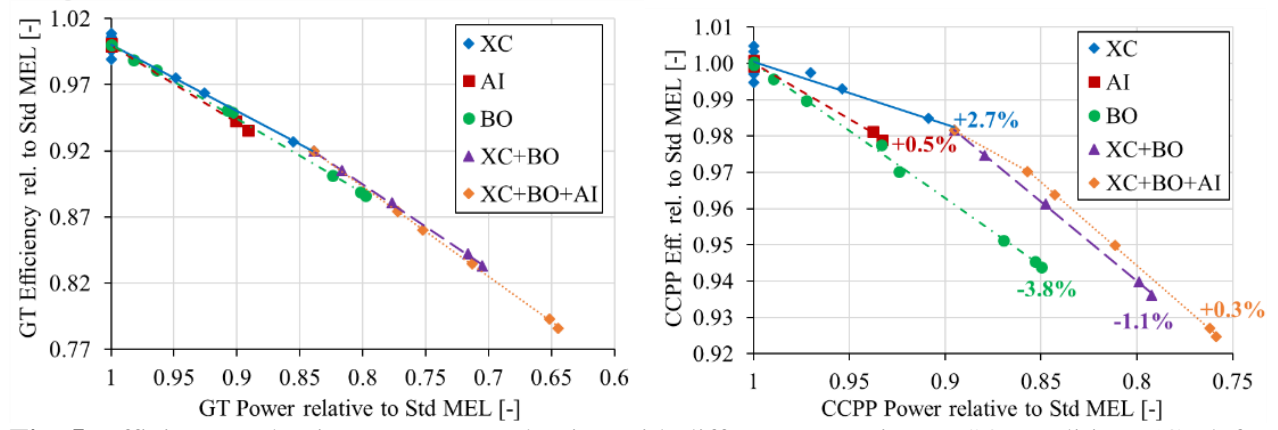

Fig. 5. Efficiency reduction Vs power reduction with different strategies at ISO conditions (GT left, CCPP right) with respect to MEL reference condition.

The situation changes looking at the CCPP performance in Fig.5 (right), because the second preferred option after IGV XC becomes the AI activation instead of BO opening. All the three strategies reduce the GT pressure ratio with respect to standard MEL because less air flows into the turbine so, with the turbine inlet temperature being fixed for emissions, the expander outlet temperature would be higher and improve WSC efficiency. In the case of BO opening, the flow exits from the last turbine blade hotter, but then a big cold flow is discharged in the middle of the turbine diffuser, cooling down the exhaust gas and strongly reducing the steam cycle efficiency and consequently the CCPP one.

According to the previous considerations, MEL reduction is a matter of compressor stability, but there are also other limitations depending on GT hardware and overall plant capabilities. For example, if the target is to reduce the CCPP's MEL through IGV XC, the GT outlet temperature cannot increase indefinitely because the HRSG has been sized on a maximum temperature level. If the HRSG limit is reached but there is still margin on compressor stability, the BO opening could help to overcome this problem because it further 
reduces MEL by decreasing the turbine outlet temperature. Similarly, with deep IGV XC the ice formation on the first stage can occur before the compressor stability limit is reached. In this case, the AI activation can help to solve the ice problem and enables to further close the IGV to reduce MEL as much as possible. Another technique for MEL reduction which is not treated here is the CO catalyst application. In this case, the GT load would be further decreased because the combustor could operate at lower flame temperature, leading to a turbine exhaust temperature reduction too.

Fig. 5 also shows the theoretical effect of the combined use of $\mathrm{XC}+\mathrm{BO}$ and $\mathrm{XC}+\mathrm{BO}+\mathrm{AI}$ to reduce the above-mentioned risks. The additional simulations have been performed starting from the deepest IGV XC (being XC the best solution to improve MEL), but the GT operability in such conditions has to be confirmed by further dedicated combustor and compressor investigation and checked versus the plant operability. Comparing the orange and purple line, the AI has negative effect on GT efficiency but positive on CCPP efficiency as already mentioned. Looking at the text boxes in Fig. 5 (right), the $\mathrm{XC}+\mathrm{BO}+\mathrm{AI}$ solution basically restores the standard MEL outlet temperature, while the $\mathrm{XC}+\mathrm{BO}$ solution resulted in a lower temperature with respect to standard MEL, so they are safe from the HRSG point of view. According to Fig. 5, the theoretical gain in terms of GT MEL reduction obtainable superimposing $\mathrm{BO}$ to $\mathrm{XC}$ is more than $29 \%$ (>20\% in CCPP), in case of BO and AI superimposed to $\mathrm{XC}$ the theoretical gain becomes more than $35 \%$ (>24\% in CCPP).

\section{Conclusions}

Three different options have been analyzed to enhance power plants turndown capability. A calibrated 2D TF full compressor model has been developed and applied to assess the impact on compressor performance and stability of BO extraction. The numerical results show that the last stator row limits the overall stability of the compressor and hence the maximum possible extraction. For low compressor power input and highest stability of rear stages, mass-flow should be extracted at the first BO line. A validated 3D CFD full compressor model has been used to simulate IGV XC impact on compressor stability. The model results show that the limiting stages for this case are the last ones, especially at very low ambient temperature conditions. Moreover, the expansion across CB01 at standard MEL and when IGV XC is applied lowers the temperature downstream of the first rotor that may result in ice deposition on the first stage rows. Eventually, through a calibrated OD model GT and CCPP performance have been assessed. It shows that in GT cycle from the efficiency point of view the best way to lower MEL condition is: 1) XC, 2) $1^{\text {st }} \mathrm{BO}$ opening 3) AI. While, as far as the CCPP is concerned the ranking is: 1) XC 2) AI 3) $1^{\text {st }} \mathrm{BO}$ opening. However, in order to mitigate the drawbacks of the 3 options, when separately applied, as well as to lower even more MEL condition, the combined (and tuned) use of these 3 strategies is promising if allowed by GT and plant limitations/operability.

These research's results are part of the TURBO-REFLEX project, which has received funding from the European Union's Horizon 2020 research and innovation programme under grant agreement No 764545 .

\section{References}

1. IEA, "WEO-2018-Executive summary-English-IEA Web store," (2018). Available: https://webstore.iea.org/download/summary/190?fileName=English-WEO-2018$\underline{\text { ES.pdf }}$ 
2. C. O. Ojo, C. Schwille, A. Nemet, T. Zierer, M. Nicklas, "Optimization of Anti-Icing Limits for Alstom Gas Turbines Based on Theory of Ice Formation," GT2009-59232, Proc. of ASME Turbo Expo 2009 (2009)

3. F. Sisto, "AGARD Manual on Aeroelasticity in Axial-Flow Turbomachines," vol. 1, ch.1,7 (AGARD, 7 Rue Ancelle, 92200 Neuilly sur Seine, France, 1987)

4. A. Sorce, "Solutions for Combined Cycle Flexibility," (2016). Available: http://www.tpg.unige.it/TPG/wp-content/uploads/2016/12/Seminario_CombinedCycle-Flexibility-Solution_12_12_2016-1.pdf

5. E. Mendoza, T. Lin, X. Jiang, "Reduction of Gas Turbine Exhaust Temperature Limit due to HRSG Limitations by Change on Control Curve to Optimize Plant Operation and Performance," GT2015-43784, Proc. of ASME Turbo Expo 2015 (2015)

6. M. Schnoes, C. Voß, E. Nicke, "Design Optimization of a Multi-Stage Axial Compressor Using Throughflow and a Database of Optimal Airfoils," J. of the GPPS, pp. 516-528 (2018)

7. A. Arnone, "Viscous Analysis of Three-Dimensional Rotor Flow Using a Multigrid Method," J. Turbomach., vol. 116, n. 3, pp. 435-445 (1994)

8. D. C. Wilcox, Turbulence Modeling for CFD, 2nd ed. (DCW Ind. Inc., La Cañada, CA, USA, 1998)

9. P. Boncinelli, F. Rubechini, A. Arnone, M. Cecconi, C. Cortese, "Real Gas Effects in Turbomachinery Flows: a CFD Model for Fast Computations," GT2003-38101, Proc. of ASME Turbo Expo 2003 (2003)

10. L. Cozzi, "Advanced CFD Modelling of Multi-Stage Axial Compressors," PhD Thesis, Firenze (2019) 